\title{
Comment les mycobactéries résistent à l'isoniazide
}

La tuberculose est responsable du quart des décès de l'adulte dans les pays en voie de développement, et la plus grande susceptibilité à la maladie, accompagnée d'une mortalité accrue, chez les sujets infectés par le virus de l'immunodéficience humaine, ne peut qu'aggraver la situation. En outre, l'émergence de souches de Mycobacterium tuberculosis multirésistantes aux antituberculeux a entraîné des épidémies mortelles dans de nombreux pays dont les États-Unis $\left(m / s n^{\circ} 11\right.$, vol. 9 p. 1279). L'acide hydrazide isonicotinique (isoniazide, INH), utilisé comme antituberculeux depuis 1952, reste la base du traitement, bien que son mécanisme d'action précis soit inconnu. Les mutants résistants à l'INH représentent actuellement jusqu'à $26 \%$ des isolats de $M$. tuberculosis dans certaines villes américaines. Certains de ces mutants sont associés à une perte de l'activité catalase, et des délétions dans le gène catalase-peroxydase (katG) ont été rapportées chez des souches de M. tuberculosis résistantes à l'INH [1]. En outre, le transfert du gène $k a t G$ d'une souche de $M$. tuberculosis sensible à l'INH confère cette sensibilité à des souches résistantes de $M$. segmatis et M.tuberculosis. Le mécanisme d'action proposé est que la catalase-peroxydase convertirait l'INH en une substance métaboliquement active [1]. Toutefois, seuls $10 \%$ à $25 \%$ des isolats résistants à l'INH provenant de New York et San Francisco sont dépourvus d'activité catalase, ce qui indique que la résistance à l'INH peut être due à d'autres facteurs. La résistance aux antibiotiques peut être causée par de nombreux mécanismes, incluant des mutations dans la cible du médicament qui en réduisent la fixation, ou des mutations qui conduisent à une augmentation de la production de la cible. Une équipe américanonéo-zélandaise vient de montrer que ces deux types de mécanismes s'appliquent aussi aux souches résistantes de mycobactéries [2]. Ils ont identifié un gène codant pour une cible de l'INH et de l'éthionamide (ETH, un analogue structural de l'INH utilisé comme antituberculeux d'appoint). Les chercheurs ont transformé des souches de $M$. segmatis sensibles à l'INH à l'aide de vecteurs navettes cosmidiques contenant une banque génomique issue, soit de souches de $M$. segmatis, soit de souches de M.tuberculosis, toutes deux résistantes à l'INH et à l'ETH. Ils ont obtenu des clones transformés de $M$. segmatis devenus résistants à l'INH et l'ETH. Les études de sous-clonage ont montré que le fragment d'ADN nécessaire pour conférer la résistance était un gène dénommé inhA, codant pour une protéine de $32 \mathrm{kDa}$. En outre, le gène inhA sauvage confère la résistance à l'INH et l'ETH quand il est transféré en multicopies à des souches sensibles de $M$. segmentis et $M$. bovis BCG. La structure primaire des protéines InhA de $M$. tuberculosis, $M$. bovis et $M$. segmentis, prédite par leur gène, a une grande similitude avec celle des protéines EnvM de Salmonella typhimurium et Escherichia coli $(40 \%$ d'identité sur 203 aminoacides). Étant donné que EnvM est impliquée dans la biosynthèse des acides gras, on peut aisément imaginer que InhA jouerait un rôle dans la biosynthèse des acides mycoliques, les acides gras à longue chaîne caractéristiques de la paroi des mycobactéries, et qu'elle serait la première cible de l'action de l'INH et de l'ETH. L'étude de la protéine InhA chez des souches de $M$. bovis et $M$. segmentis résistantes à l'INH a révélé une unique substitution d'une sérine en alanine en position 94 par rapport à la protéine des souches sensibles.

Plusieurs groupes ont maintenant testé des souches cliniques de M. tuberculosis résistantes à l'lNH, à la recherche de mutations dans la région codante du gène $i n h A$ et en ont trouvé [3]. Toutefois, on peut imaginer qu'il existe des mutations en dehors de la région codante entraînant une surexpression de InhA (par exemple dans le promoteur de inhA ou dans un gène de régulation) avec comme conséquence une résistance à l'INH.

S'il se confirme que les altérations de inhA et katG rendent compte de la majorité des résistances à l'INH, les cliniciens auront bientôt à leur disposition un test basé sur la technique PCR qui leur permettra de déterminer rapidement si leur patient est infecté par une souche résistante et donc de prendre les mesures nécessaires. En outre, la découverte de ces deux gènes va donner un souffle nouveau au développement d'agents antituberculeux.

E.D

1. Zhang Y, Heym B, Allen D, Young D, Cole $\mathrm{S}$. The catalase peroxidase gene and isoniazid resistance of Mycobacterium tuberculosis. Nature 1992 ; 358 : 591-3.

2. Banerjee A, Dubnau E, Quemard A, Balasubramanian V, Um KS, Wilson T, Collins S, de Iisle (; Jacobs WR. inhA, a gene encoding a target for isoniazid and ethionamide in Mycobacterium tuberculosis. Science 1994; 263 . 227-30.

3. Travis J. Unveiling a tuberculosis drug target. Science 1994 ; 263 : 172 